\title{
A Novel Two-step Method for CT Reconstruction
}

\author{
Michael Felsberg \\ Computer Vision Laboratory, Dept. EE, Linköping University, Sweden \\ mfe@isy.liu.se
}

\begin{abstract}
In this paper we address the parallel beam $2 \mathrm{D}$ computer tomography reconstruction. The proposed method belongs to the field of analytic reconstruction methods and is compared to several methods known in the field, among other the two-step Hilbert-transform method. In contrast to the latter, the derivative data is multiplied with an orientation vector and the Hilbert transform is replaced with the Riesz transform. Experimental results show that the new method is superior to established ones concerning aliasing, noise, and DC errors.
\end{abstract}

\section{Introduction}

Given a number of line projections of an object, we want to reconstruct its density. This type of problem typically occurs in context of parallel beam computer tomography $(\mathrm{CT})$ reconstruction. For this paper, we consider a simplified 2D setting neglecting a number of physical phenomena (Fig. 1a). The measuring unit consists of a source emitting parallel rays and a detector measuring the intensity of each incoming ray for each position $s$. The object between source and detector attenuates the rays depending on its density. The problem to solve for $\mathrm{CT}$ reconstruction is to compute the $2 \mathrm{D}$ density distribution of the object from the set of its line projections at different rotation angles $\theta$.

The object is located at the origin of the $2 \mathrm{D}$ coordinate system $(x, y)^{T}$. The coordinate system of the measuring unit $(s, t)^{T}$ and is rotated by $\theta$ around the

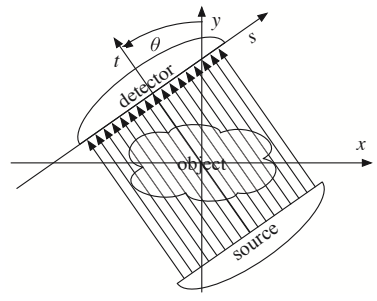

(a)

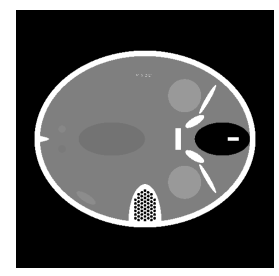

(b)

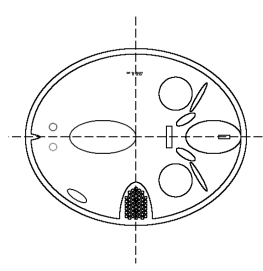

(c)

Fig. 1. (a) 2D geometry for the projections. Note that the $s$-axis was moved to the detector in order to visualize that $s$ is at the same time the position on the detector. (b) Phantom used for experiments. (c) Edge map of phantom in (b) 
Fig. 2. Different options for analytic linear reconstruction. (a) ramp-filtered backprojection. (b) two-step reconstruction using the Hilbert transform [2]. (c) post-filtered back-projection [3]. (d) three-step Hilbert method [4]. Proposed method

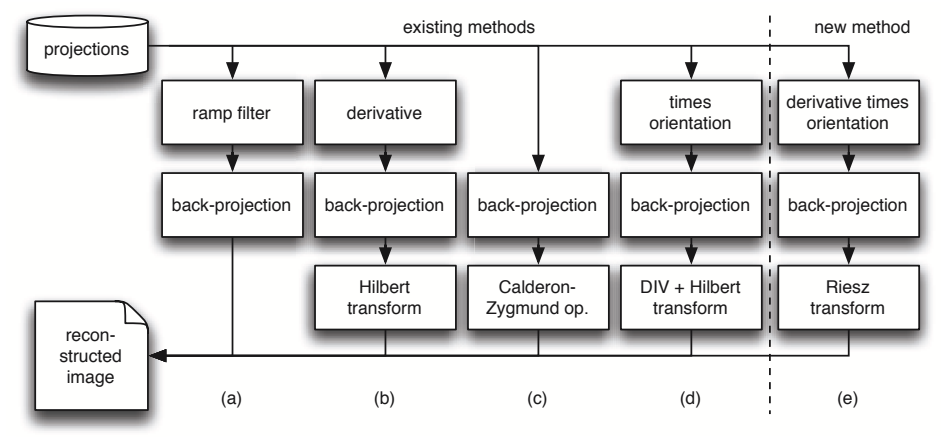

origin. The Radon transform [1] is obtained by integrating the object density $f(x, y)$ along $t: p_{\theta}(s)=\int_{-\infty}^{\infty} f(x, y) d t=\int_{-\infty}^{\infty} f(s \cos \theta-t \sin \theta, s \sin \theta+t \cos \theta) d t$.

Various analytic methods for reconstruction have been suggested in the literature. We have summarized the relevant methods in Fig. 2. Starting point for all considered approaches is the projection slice theorem [5] resulting in $f(x, y)=\int_{-\infty}^{\infty} \int_{0}^{\pi} P_{\theta}(m) \exp (i 2 \pi m(\cos \theta x+\sin \theta y))|m| d \theta d m$, where $P_{\theta}(m)$ is the 1D Fourier transform ${ }^{1}$ of $p_{\theta}(s)$. For what follows, we define the back-projection operator as $\mathcal{B}\left\{g_{\theta}\right\}=\int_{0}^{\pi} g_{\theta}(\cos \theta x+\sin \theta y) d \theta$, where $g_{\theta}$ is a family of $1 \mathrm{D}$ functions parametrized by $\theta$.

For the ramp-filtered back-projection, $p_{\theta}$ is filtered with the 1D ramp filter, corresponding to $|m|$, and then back-projected, cf. Fig. 2 (a). Denoting the ramp-filtered projection as $p_{\theta}^{r}$, we obtain $f(x, y)=\mathcal{B}\left\{p_{\theta}^{r}\right\}$. For the postfiltered back-projection, the back-projected image is filtered with the $2 \mathrm{D}$ ramp filter $\sqrt{u^{2}+v^{2}}$, the Calderon-Zygmund operator [3], cf. Fig. 2 (c): $f(x, y)=$ $\left[\int_{-\infty}^{\infty} \int_{-\infty}^{\infty} \sqrt{u^{2}+v^{2}} \exp (i 2 \pi(u x+v y)) d u d v\right] * \mathcal{B}\left\{p_{\theta}\right\}$.

For the two-step reconstruction using the Hilbert transform, which goes back to the original work of Radon [1], the term $|m|$ is split into two factors $|m|=(i m)(-i \operatorname{sign}(m))$, where the first factor corresponds to the derivative in the spatial domain (up to a factor $2 \pi$ ) and the second factor is the Hilbert transform. The latter is computed after the back-projection [2], cf. Fig. 2 (b): $f(x, y)=2 \pi \mathcal{H}\left\{\mathcal{B}\left\{p_{\theta}^{\prime}\right\}\right\}$, where $\mathcal{H}$ is the Hilbert transform operator acting in an arbitrary direction ${ }^{2}$ and $p_{\theta}^{\prime}$ denotes the derivative of the projection. For the three-step Hilbert method, the projection data is multiplied with the orientation vector before back-projection and the Hilbert transform is computed on the divergence of back-projected vector field [4], cf. Fig. 2 (d): $f(x, y)=$ $2 \pi \mathcal{H}_{y}\left\{\left\langle\nabla ; \mathcal{B}\left\{(\cos \theta, \sin \theta)^{T} p_{\theta}\right\}\right\rangle\right\}$, where $\langle\cdot ; \cdot\rangle$ denotes the scalar product and $\nabla=\left(\partial_{x}, \partial_{y}\right)^{T}$ is the gradient operator.

${ }_{1}^{1}$ D Fourier kernel: $\exp (-i 2 \pi m s) ; 2 \mathrm{D}$ Fourier kernel: $\exp (-i 2 \pi(u x+v y))$

${ }^{2}$ For the sake of simplicity we apply the vertical transform in what follows. 


\section{Materials and Methods}

For the novel reconstruction method, we split the Calderon-Zygmund operator into two parts:

$$
\sqrt{u^{2}+v^{2}}=\left\langle i(u, v)^{T} ;-i \frac{(u, v)^{T}}{\sqrt{u^{2}+v^{2}}}\right\rangle=\left\langle i(u, v)^{T} ; R^{T}(u, v)\right\rangle
$$

where the left factor corresponds to the 2D gradient (up to a factor of $2 \pi$ ) and the right factor corresponds to the Riesz transform, a rotation-invariant generalization of the Hilbert transform [6]. The method of rotation [6] results in a relation between the 1D Hilbert transform and the 2D Riesz transform [7], which can be applied in this case for expressing the $2 \mathrm{D}$ gradient in the projection domain as $(\cos \theta, \sin \theta)^{T} p_{\theta}^{\prime}(s)$, leading to the final reconstruction formula

$$
f(x, y)=2 \pi \mathcal{R}\left\{\mathcal{B}\left\{(\cos \theta, \sin \theta)^{T} p_{\theta}^{\prime}\right\}\right\},
$$

where $\mathcal{R}$ is the Riesz transform operator. The latter is defined by $\sqrt{-\Delta}=\langle\nabla \mid \mathcal{R}\rangle$, where $\Delta=\langle\nabla \mid \nabla\rangle=\partial_{x} \partial_{x}+\partial_{y} \partial_{y}$ is the Laplace operator. The Riesz operator in the reconstruction (2) should be interpreted as a scalar product, i.e., the respectively first and second component of $\mathcal{R}$ is applied to the first and second component of the integral.

For periodic boundary conditions, $\mathcal{R}$ corresponds to the frequency response $R(u, v)$. For Neumann boundary conditions, the solution has been given in [8]. For the here considered problem, the ideal setting would be to restrict the computation to a circular domain, but practical considerations require a rectangular domain. The reasons are twofold: a) images are typically rectangular shaped and b) the rectangular domain solution can be computed by FFT algorithms.

Hence, we require the reconstruction to be zero outside a rectangular domain, i.e., the reconstruction has compact support. Consequently, all projections have a finite support and so do the derivatives. Applying the back projection to the projection derivatives, however, results in a $2 \mathrm{D}$ function with infinite support. This can easily be verified by either considering the projection geometry or the non-locality of the Riesz kernel. If we back-project onto a finite image, we cut of the tails of the filtered gradient image. Hence, our task is to compute the finite Riesz transform of an infinite gradient signal which is truncated, knowing that the result is zero outside the considered domain.

Assume that $b(x, y)=\mathcal{B}\left\{(\cos \theta, \sin \theta)^{T} p_{\theta}^{\prime}\right\}$ is the back-projected oriented derivative on the domain $(x, y) \in\left(0 ; x_{\max }\right) \times\left(0 ; y_{\max }\right)$. Define the modified signal $b_{m}(x, y)$ on the domain $(x, y) \in\left(-x_{\max } ; x_{\max }\right) \times\left(-y_{\max } ; y_{\max }\right)$ by

$$
b_{m}(x, y)=\left(\begin{array}{cc}
\operatorname{sign}(y) & 0 \\
0 & \operatorname{sign}(x)
\end{array}\right) b(|x|,|y|)
$$

i.e., the components of $b$ are mirrored at the coordinate axes, with anti-symmetry with respect to the $y$ - and $x$-axis, respectively. As a result, the signal tails which should exist in the back-projected image $b$ compensate each other and a good approximation of the Riesz transform with finite data becomes possible. 
The Riesz transform of the signal $b(x, y)$ under the side condition of zero continuation outside of $\left(0 ; x_{\max }\right) \times\left(0 ; y_{\max }\right)$ is computed using the DFT and the frequency response of the Riesz transform:

$$
\mathcal{R}\{b\}=\mathrm{iDFT}\left\langle-i \frac{(u, v)^{T}}{\sqrt{u^{2}+v^{2}}} \mid \operatorname{DFT}\left\{b_{m}\right\}\right\rangle
$$

The new method described in the previous paragraphs has slightly higher computational complexity than ramp-filtered back-projection, post-filtered backprojection, and the two-step Hilbert method. Similar to the three-step Hilbert method, two images need to be back-projected and back-projection is the computationally most demanding step in all cases. The higher complexity can be justified by three significant advantages, some of which are shared with some of the methods from the literature, but not with a single one:

- Aliasing. The two-step Hilbert method and the Riesz-method use DC-free projection data, which suppresses DC aliasing altogether without any particular efforts for the design of the interpolation filter.

- SNR. The Riesz-method shows (together with the three-step Hilbert method) significant lower noise levels since both methods reconstruct orientation dependent and noise is typically unoriented.

- DC estimation. In contrast to the two Hilbert-methods, the Riesz-method only needs to be complemented by a single DC estimate, whereas the Hilbertmethods need an additional DC estimate for each column of the reconstructed image.

The experiment that we use to verify these properties is based on noisy projections from the phantom in Fig. 1 (b). For verifying the alias components visually, we apply a Prewitt edge detector to the resulting images. The SNR is measured along the dashed lines in Fig. 1 (c). The DC estimation problematic is verified by visually inspecting reconstructed images without edge detection.

\section{$3 \quad$ Results}

The reconstruction results can be found in Fig. 3 .

\section{Discussion}

Comparing the results in Fig. 3 with respect to aliasing, SNR, and DC estimation, we draw the following conclusions:

- Aliasing. As claimed before, the methods which are back-projecting a derivative do not show the aliasing artefacts that are visible in the other methods, cf. (b) and (d) vs. (a) and (c).

- SNR. The noise level in the methods without orientation vector is more than $10 \%$ higher than for the other methods, cf. (a) and (d) vs. (b) and (c). 
Fig. 3. Reconstructed densities. Note that the intensity range in (e) is amplified compared to (f) in order to show the presence of the weaker structures

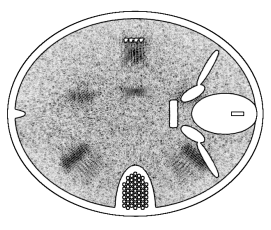

(a) ramp-filtered

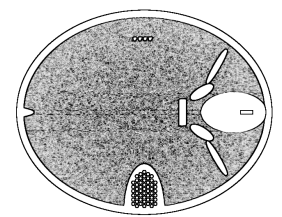

(d) two-step Hilbert

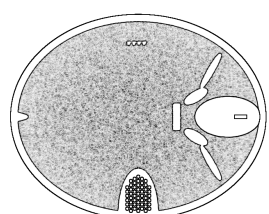

(b) Riesz

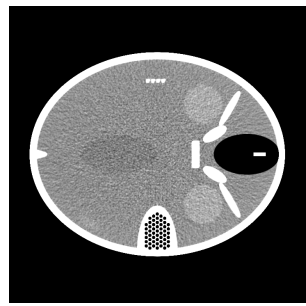

(e) Riesz (intensity)

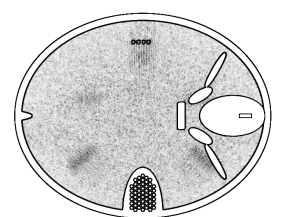

(c) three-step Hilbert

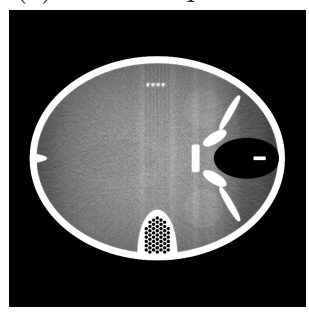

(f) three-step Hilbert (intensity)

- DC estimation. The DC estimation for the Hilbert-based methods is fairly tricky without prior knowledge about the object, cf. (f) vs. (e).

Summarising the experimental results, we conclude that the Riesz method combines all positive properties of the compared methods in a single method.

\section{References}

1. Radon J. On the determination of functions from their integral values along certain manifolds. IEEE Trans Med Imaging. 1986;5:170-6.

2. Noo F, Clackdoyle R, Pack JD. A two-step Hilbert transform method for 2D image reconstruction. Phys Med Biol. 2004;49:3903-23.

3. Solmon DC. Asymptotic formulas for the dual Radon transform and applications. Math Z. 1987;195:321-43.

4. Zeng GL. Image reconstruction via the finite Hilbert transform of the derivative of the backprojection. Med Phys. 2007;34:2837-43.

5. Bracewell RN. Two-Dimensional Imaging. Prentice Hall Signal Processing Series. Prentice Hall, Englewood Cliffs; 1995.

6. Stein EM, Weiss G. Introduction to Fourier Analysis on Euclidean Spaces. Princeton University Press, New Jersey; 1971.

7. Felsberg M, Sommer G. The monogenic signal. IEEE Trans Signal Process. 2001;49:3136-44.

8. Felsberg M, Duits R, Florack L. The monogenic scale space on a rectangular domain and its features. Int J Comput Vis. 2005;64. 